\title{
T cell apoptosis and reactive oxygen species
}

\author{
David A. Hildeman, ${ }^{1}$ Thomas Mitchell, ${ }^{2}$ John Kappler, ${ }^{3,4,5,6}$ and Philippa Marrack $3,4,5,6,7$ \\ ${ }^{1}$ Division of Immunobiology, Children's Hospital Medical Center, Cincinnati, Ohio, USA \\ ${ }^{2}$ Institute for Cellular Therapeutics, University of Louisville School of Medicine, Louisville, Kentucky, USA \\ ${ }^{3}$ Howard Hughes Medical Institute, and \\ ${ }^{4}$ Department of Medicine, National Jewish Medical and Research Center, Denver, Colorado, USA \\ ${ }^{5}$ Department of Pharmacology, \\ ${ }^{6}$ Department of Integrated Immunology, and \\ ${ }^{7}$ Department of Biochemistry and Molecular Genetics, University of Colorado Health Sciences Center, Denver, Colorado, USA \\ J. Clin. Invest. 111:575-581 (2003). doi:10.1172/JCI200318007.
}

$\mathrm{T}$ cells respond to antigen stimulation through a process of activation, division, and differentiation to generate a large pool of activated effector $\mathrm{T}$ cells. Regulated induction of apoptosis of these expanded T cells avoids several problems for the host, including increased metabolic cost, disruption of lymphoid homeostasis, and predisposition to autoimmunity and lymphoid neoplasia. On the other hand, it is essential that some activated $\mathrm{T}$ cells survive apoptotic death to become memory $\mathrm{T}$ cells. Thus, an understanding of why most expanded $\mathrm{T}$ cells die, while some survive, will shed light on how autoimmunity and lymphoid cancer are prevented, and how immunity is acquired. Recent studies have identified the molecular details of this apoptotic process that operate in vivo. It is now becoming clear that two separate pathways - activationinduced cell death (AICD) and activated T cellautonomous death (ACAD) - control the fate of antigen-specific $\mathrm{T}$ cells. Interestingly, reactive oxygen species (ROS) can control both pathways through reciprocal modulation of the main effector molecules FasL and Bcl-2. This review will focus on the role played by ROS in the determination of activated $\mathrm{T}$ cell fate.

\section{The kinetics of $\mathrm{T}$ cell responses in vivo}

The endogenous $\mathrm{T}$ cell response to many different antigens follows a remarkably similar pattern. Following antigen administration, $T$ cells proliferate vigorously and reach a peak of expansion (Figure 1). This

\footnotetext{
Address correspondence to: David A. Hildeman, Department of Pediatrics, Division of Immunobiology, MLC 7038, Children's Hospital Medical Center, 3333 Burnet Avenue, Cincinnati, Ohio 45229, USA. Phone: (513) 636-3923; Fax: (513) 636-5355; E-mail: David.Hildeman@chmcc.org.

Conflict of interest: The authors have declared that no conflict of interest exists.

Nonstandard abbreviations used: activation-induced cell death (AICD); activated T cell autonomous death (ACAD); reactive oxygen species (ROS); superoxide dismutase (SOD); manganese (III) tetrakis 5, 10, 15, 20 benzoic acid porphyrin (MnTBAP); $\mathrm{T}$ cell receptor (TCR); nuclear factor of activated T cells (NFAT); Bcl-2 homology (BH); cAMP response element-binding protein (CREB).
}

peak does not have a predetermined upper limit, since it can be further increased by certain adjuvants (i.e., LPS or stimulating antibodies to OX40, CD40, or 4$1 \mathrm{BB})$ or by manipulation of the antigen dose. However, the number of activated $T$ cells at this stage cannot, of course, exceed the capacity of the animal to support them. It is thought that few antigen-specific $T$ cells die during the expansion phase, because of the obvious impairment of expansion that this would cause, although little has been done to explore this possibility. After the $\mathrm{T}$ cells reach their peak of expansion, their numbers begin to decline rather rapidly, a process that has been referred to as contraction (1). Recent reports have shown that during this decline, some of the $\mathrm{T}$ cells appear to migrate to nonlymphoid tissues and to reside there as effector memory $\mathrm{T}$ cells $(2,3)$. However, the numbers of $\mathrm{T}$ cells that migrate to nonlymphoid tissues cannot account for the total number of cells that exist at the peak of the response, and most of the activated cells disappear because they apoptose (4). Recent research has uncovered two sepa-

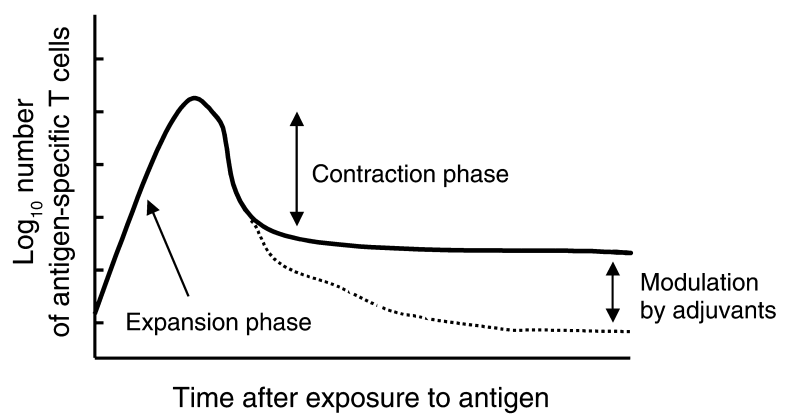

Figure 1

Graph of the kinetics of the T cell response to antigenic stimulation in vivo. Following stimulation with antigen, antigen-specific $T$ cells proliferate vigorously to reach peak numbers during the expansion phase. They then decline in number rapidly, a process referred to as the contraction phase. Importantly, both the peak-expansion phase and the contraction phase can be independently modulated by stimulators of innate immunity (adjuvants) $(53,54)$. 
rate pathways responsible for the death of activated, antigen-specific T cells in vivo. One of these pathways, AICD, is driven by signals delivered exogenously to the cell. The other pathway, ACAD, is driven by signals that are intrinsic to the activated $\mathrm{T}$ cell.

\section{Activation increases the levels of ROS in T cells: ROS may play a role in activated $\mathrm{T}$ cell death}

Activation increases the amount of ROS in T cells (5-7), although it is unclear how these extra ROS are created. T cells lack the conventional NADPH oxidase enzymes used by granulocytes for oxidative bursts. However, other mechanisms for producing ROS have been described and might occur in T cells. One such mechanism could be driven by the increased demands for ATP production imposed on T cells by their conversion from a resting condition to the state of rapid cell division that accompanies activation. This is exemplified by experiments in which thymocytes are stimulated with phorbol myristate acetate and ionomycin, leading to rapid glucose consumption followed by increased oxidative phosphorylation and a subsequent increase in ROS production (8). This production of ROS probably occurs as a consequence of direct interaction of electrons shed from the respiratory chain with molecular oxygen, resulting in the formation of superoxide (9). Increased demands on mitochondrial electron transport for energy can therefore lead to the increased levels of superoxide within cells. For T cells this is evidenced by increased alkalinization of the cell cytosol (which is indicative of increased respiratory activity) rather than increased acidification (which would be evidence of increased glycolytic activity) (10). Thus, mitochondria can be a major source of ROS within cells, and increased energy demands, such as those seen with rapid $\mathrm{T}$ cell proliferation, can increase levels of mitochondrially derived ROS.

One group has reported that ROS levels increase within 15 minutes of $\mathrm{T}$ cell activation, a time that is too short for production of ROS via increased mitochondrial activity (6). Thus, it may be that reactions that are more immediate than increased mitochondrial activity are responsible for at least the initial increases in ROS levels in activated T cells. A wellknown pathway for certain receptors to induce ROS involves Ras/Rac/PI3K signaling to activate NADPH oxidase enzymes. However, as mentioned previously, because $T$ cells do not express these enzymes, it is unlikely that this pathway plays a role.

Another putative source of ROS might be peroxisomal enzymes; however, no studies of the role peroxisomes play in the generation of ROS in T cells have been reported. Interestingly, agonists of PPAR $\gamma$ cause significant apoptosis of activated T cells (11), and although the authors of this study did not examine ROS generation by this agonist, a recent report has shown that agonists of PPAR $\gamma$ can induce rapid superoxide generation in myoblast cells (12).

Some have suggested that bystander neutrophils might be the source of the increased ROS levels in activated $T$ cells. In this instance, it is postulated that acti- vated neutrophils produce the ROS and that these ROS then diffuse into neighboring $T$ cells (13). However, under most circumstances the ROS are produced by the $T$ cells themselves (5-7).

Regardless of their origin, the detoxification of these ROS by antioxidant enzymes can influence their levels as well. For example, superoxide dismutases (SODs) convert two superoxide molecules into one oxygen and one hydrogen peroxide $\left(\mathrm{H}_{2} \mathrm{O}_{2}\right)$ molecule (9). In turn, $\mathrm{H}_{2} \mathrm{O}_{2}$ is detoxified by glutathione peroxidase and/or catalase (9). While SOD converts superoxide into another toxic ROS, $\mathrm{H}_{2} \mathrm{O}_{2}$, it is important to note that the reaction of superoxide with iron- and sulfur-containing proteins such as aconitase produces $\mathrm{H}_{2} \mathrm{O}_{2}$ as well (14). Furthermore, dismutation of superoxide radicals by SOD produces half the amount of $\mathrm{H}_{2} \mathrm{O}_{2}$ produced by direct interaction of superoxide with oxidizable molecules such as aconitase and prevents the release of damaging iron $(14,15)$. In addition, the levels and/or activities of glutathione peroxidase and/or catalase are critical, because interactions of $\mathrm{H}_{2} \mathrm{O}_{2}$ with transition metals can lead to the highly reactive hydroxyl radical via Fenton or Haber-Weiss chemistry (9). Thus, the outcome of ROS damage is ultimately dependent upon the levels and type of ROS made, both of which are dependent upon the levels and activities of endogenous antioxidant enzymes. Unfortunately, little work has been done to examine the activities of the endogenous antioxidant enzymes before and during $\mathrm{T}$ cell activation in vivo. One report has shown that $\mathrm{T}$ cells are deficient in both their ability to synthesize and their ability to take up glutathione precursors, possibly causing decreases in glutathione peroxidase activity (16). Clearly, much more work needs to be done to determine the relationship between $\mathrm{T}$ cell activation and activities of endogenous antioxidant enzymes.

Despite the fact that very little is known about the molecular events that lead to ROS production and ROS detoxification within $T$ cells, several studies have shown that activated $\mathrm{T}$ cells can be killed by these entities $(6,7)$. We demonstrated that $T$ cells that have been activated in vivo and isolated just before they would have started to die in the animal, die very rapidly in culture. This rapid death is markedly slowed by addition of a synthetic, catalytic manganese superoxide dismutase mimetic, manganese (III) tetrakis 5, 10, 15, 20 benzoic acid porphyrin (MnTBAP) (7). Results of this nature have led to interest in the roles that ROS might pay in the various pathways of $\mathrm{T}$ cell death, as discussed below.

\section{AICD}

Ashwell and colleagues made the initial observation that, instead of causing proliferation, $\mathrm{T}$ cell receptor (TCR) stimulation of T cell hybridomas resulted in decreased growth and a block in the cell cycle (17). Green's group further investigated this phenomenon and showed that anti-CD3-treated $\mathrm{T}$ cell hybridomas and thymocytes underwent significant apoptosis, a process they referred to as AICD (18). Since these initial in vitro observations, the molecular details of 
AICD have been worked out. It is generally well accepted that AICD is mediated predominantly by signaling through the death receptor Fas and, in certain instances, by signaling through TNF- $\alpha$ receptors. Mice with functional defects in either Fas or FasL ( $l p r$ and $g l d$, respectively) were used to determine whether AICD plays a role in termination of $\mathrm{T}$ cell responses in vivo. A few reports showed that $T$ cell responses were prolonged in lpr or gld mice, leading many in the field to believe that Fas signaling is required for the death of all activated $\mathrm{T}$ cells in vivo $(19,20)$.

\section{Molecular control of AICD}

In vitro activation of $\mathrm{T}$ cells results in increased expression of FasL, which, upon binding to and oligomerization of Fas, results in the direct activation of caspase enzymes via their recruitment to a deathinducing signaling complex (DISC) (reviewed in ref. 20). Once these upstream caspases are activated in the DISC, the apoptotic program is initiated, and downstream caspases and other endogenous substrates are then proteolytically cleaved, resulting in the demise of the cell by classical apoptosis.

In theory, T cell death caused by AICD could be controlled in several ways: by control of Fas expression, by control over the pathways downstream of Fas engagement that lead to cell death, or by control of FasL expression. While Fas can be upregulated following $\mathrm{T}$ cell activation, $\mathrm{T}$ cells express significant amounts of Fas to begin with, so expression of this molecule is probably not a controlling factor (21).

Modulation of Fas signaling after engagement by FasL is mainly achieved by the expression of an enzymatically inert homologue of caspase- 8 called FLIP (22). Resting primary T cells express high levels of FLIP, are resistant to AICD, and require downregulation of FLIP by IL-2 to acquire sensitivity to Fas-driven death (23). cFLIP transcription has been reported to be induced by MEK1, though it is unclear whether this is the mechanism by which resting $\mathrm{T}$ cells maintain high levels of FLIP (24). Alternatively, TGF- $\beta$ may be involved, since it is known to induce FLIP, and since TGF- $\beta$-deficient mice and $T$ cells from these mice display increased sensitivity to Fas-driven $\operatorname{AICD}(25,26)$. In any case, levels of FLIP probably affect induction of AICD in cells, but a role for ROS in FLIP expression is currently not suspected.

\section{Control of FasL expression by ROS}

Along with FLIP expression, FasL expression is the major means by which AICD is controlled. Engagement of the TCR increases FasL expression on T cells (27). A fairly coherent pathway can be proposed to account for such induction, although all the pieces of the pathway have not been fully established in T cells. TCR engagement leads to increased $\mathrm{Ca}^{2+}$ levels in the cytoplasm of the cell. This may occur via two routes; one is well known and involves phospholipase $\mathrm{C} \gamma$, production of inositol 1,4,5-trisphosphate, and release of $\mathrm{Ca}^{2+}$ from intracellular stores (28). The other route may involve TCR-induced production of ROS through increased mitochondrial activity consequent to $\mathrm{T}$ cell activation. ROS, in turn, increase cytoplasmic $\mathrm{Ca}^{2+} \mathrm{lev}-$ els via pathways that are not fully understood (29).

Increased levels of intracellular $\mathrm{Ca}^{2+}$ activate the phosphatase calcineurin. Once activated, calcineurin dephosphorylates nuclear factor of activated $\mathrm{T}$ cells (NFAT) (Figure 2). Dephosphorylated NFAT translocates to the nucleus and mediates gene transcription. While active NFAT sites have been mapped within the FasL promoter, optimal NFAT-mediated regulation of FasL now appears to occur through NFAT-driven upregulation of the early growth response transcription factors Egr2 and/or Egr3 (30).

Support for the idea that ROS are intermediates in the induction of FasL after TCR engagement stems from two reports that the induction is inhibited by antioxidants $(6,31)$. One group showed that, in addition to antioxidants, inhibitors of mitochondrial function also blocked TCR-induced FasL mRNA induction, suggesting a possible role for mitochondrially derived ROS (31). They also showed that, in the absence of TCR stimulation, exogenously added $\mathrm{H}_{2} \mathrm{O}_{2}$ could induce FasL mRNA and that this was modestly inhibited by a dominant negative version of I $\mathrm{B} \alpha$. Their results suggest that, at least for Jurkat cells, $\mathrm{H}_{2} \mathrm{O}_{2}$ activation of NF- $\kappa B$ may be responsible for FasL mRNA induction. Another group more closely assessed the nature of the ROS produced very early after TCR stimulation (6). They found that both superoxide and $\mathrm{H}_{2} \mathrm{O}_{2}$ were pro-

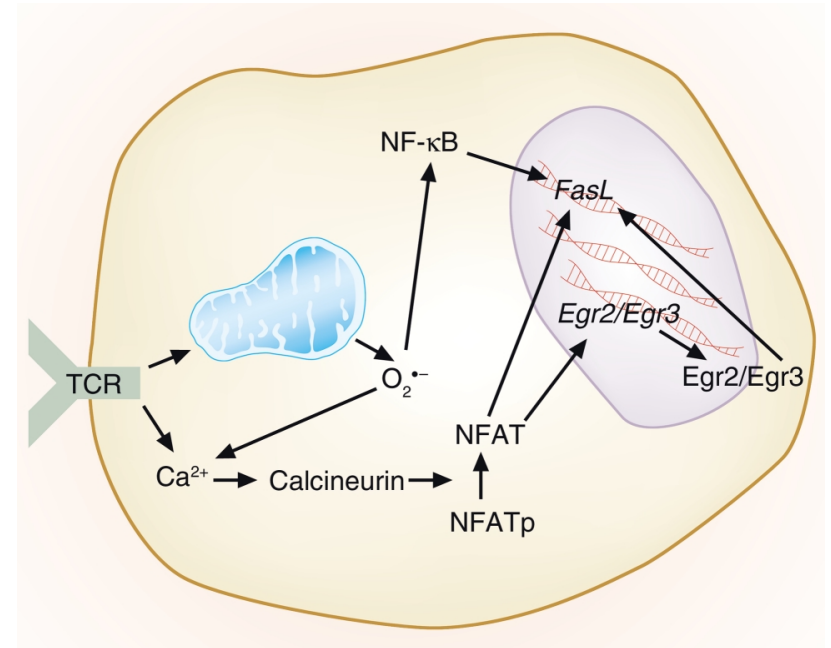

Figure 2

Control of AICD by ROS. T cell activation via TCRs leads to rapid generation of both superoxide and $\mathrm{H}_{2} \mathrm{O}_{2}$. TCR activation also leads to FasL induction. This occurs because TCR activation raises intracellular $\mathrm{Ca}^{2+}$ levels, thus activating the phosphatase calcineurin. Calcineurin dephosphorylates NFAT, and the now activated NFAT can migrate to the nucleus and contribute to Fas $L$ transcription directly via the NFAT-binding sites upstream of the gene. NFAT can also stimulate FasL transcription indirectly, by increasing transcription of the genes for the early growth response proteins Egr2 and Egr3, which in turn can themselves induce transcription of FasL. Another pathway that may be involved in FasL induction by ROS may involve signaling through $N F-\kappa B$, via activation of I $\mathrm{KB}$ by $\mathrm{H}_{2} \mathrm{O}_{2}$. NFATp, phosphorylated NFAT. 


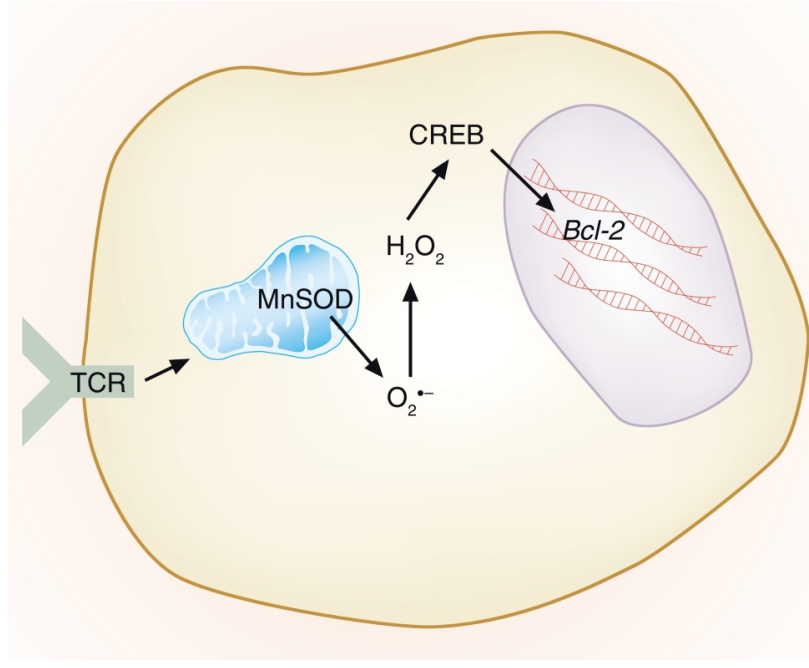

Figure 3

Control of ACAD by ROS. TCR activation leads to increased mitochondrial production of superoxide that is converted to $\mathrm{H}_{2} \mathrm{O}_{2}$ either via reactions with endogenous substrates (not shown) or via manganese superoxide dismutase (MnSOD). These ROS may have effects on various transcription factors, such as CREB, that may play a role in the downregulation of $\mathrm{Bcl}-2$ and in subsequent sensitivity of $\mathrm{T}$ cells to the effects of ACAD.

duced following TCR stimulation but suggested that superoxide and not $\mathrm{H}_{2} \mathrm{O}_{2}$ was responsible for upregulation of FasL after TCR stimulation. However, this study is confounded by the fact that the authors used electroporation as a means of introducing plasmids that encode antioxidant $\mathrm{cDNAs}$ into $\mathrm{T}$ cells, and that this process not only kills cells but also induces large amounts of ROS (32). The results of these studies point to the possibility that FasL upregulation is controlled by ROS; however, this effect is likely to be cell type-specific, since we have observed that antioxidants do not prevent FasL-driven death of DO11.10 T cell hybridomas following anti-CD3 stimulation (data not shown). Thus, while the aforementioned studies point to a possible role for ROS in controlling the induction of FasL after TCR stimulation, it will be of interest to determine where these ROS mediate their effects in the physiologic regulation of FasL following normal TCR activation of primary $\mathrm{T}$ cells.

\section{ACAD and the role of $\mathrm{Bcl}-2$-related proteins}

While Fas signaling contributes to the death of some activated $T$ cells, it certainly does not control the death of all, or even most, activated T cells in vivo, since several reports have shown that activated $T$ cells die almost equally well in the presence or absence of Fas signaling $(7,19,33,34)$.

The Fas- and TNF- $\alpha$-independent pathway for T cell death has been referred to as passive cell death or death by neglect, implying that it is mediated by cytokine or antigen withdrawal. However, we prefer to use the term ACAD (35), because the former terms are inadequate in describing what now appears to be a programmed and regulated event. This is illustrated by experiments from numerous groups (for example, refs. 1, 36) that show that $\mathrm{T}$ cell contraction occurs prior to antigen depletion, and suggest that contraction of the $T$ cell response is a preprogrammed event set in place very early after TCR stimulation in vivo, not simply the consequence of the lack of a stimulus.

Several experiments suggest that ACAD is controlled by various members of the $\mathrm{Bcl}-2$ family of proteins. This family consists of pro- and antiapoptotic members that control the fate of cells by protection or destruction of mitochondria (37). Three main classes of Bcl-2-related proteins exist. Class $1 \mathrm{Bcl}-2$-like proteins are antiapoptotic and contain Bcl-2 homology (BH) domains 1-4. Class 2 proteins are proapoptotic and Bax-like and contain $\mathrm{BH}$ domains 1-3. Class 3 proteins are included in a large family of proapoptotic $\mathrm{BH} 3$-only proteins that are expressed in a relatively tissue-specific manner. These BH3-only proteins contain a short stretch of 9 amino acids termed the $\mathrm{BH} 3$ domain but do not contain other regions of homology with $\mathrm{Bcl}-2$. The Bcl-2-like proteins function partly by interacting physically with one another. How this interaction specifically results in induction of or protection from apoptosis is still only vaguely understood. However, Thompson, and their colleagues have shown that the class 3 proteins require a class 2 protein (either Bax or Bak) to execute their apoptotic signals, at least in higher vertebrates (38).

Regardless of how these molecules interact, their induction of apoptosis involves the release of cytochrome $c$ and other apoptogenic factors from mitochondria $(37,39)$. Besides the depolarization and inactivation of the mitochondria by $\mathrm{Bcl}$-2-like proteins, the factors released by these damaged mitochondria can activate caspases in the cytosol as well as directly damage DNA in a caspase-independent manner. While T cells express several different kinds of Bcl-2-like molecules, the role they play in $\mathrm{T}$ cell apoptosis has only recently been investigated.

$\mathrm{Bcl}-2$ itself certainly plays a role in ACAD. ACAD is inhibited by increased expression of the antiapoptotic protein $\mathrm{Bcl}-2$ in $\mathrm{T}$ cells, a property that distinguishes it from the Fas-driven AICD pathway $(19,40)$. More physiologically, levels of Bcl-2 mRNA and protein drop as activated $T$ cells approach the stage at which they will die $(33,41)$. This fall in $\mathrm{Bcl}-2$ is common to all the activated cells. The few activated $T$ cells that are destined to survive also contain low levels of Bcl-2, suggesting that the signal that prevents death of surviving, memory cells acts via a pathway that does not involve Bcl-2. The idea that the decrease in $\mathrm{Bcl}-2$ contributes to ACAD is supported by experiments in which Bcl-2 levels are increased in activated $T$ cells via retroviral transduction, an event that prevents $T$ cell apoptosis both in vitro and in vivo $(33,42)$.

$\mathrm{Bcl}-2$ is not the only member of the family that affects ACAD. Recent publications have shown that the death of activated $T$ cells in vivo and in vitro and, to a certain extent, the death of thymocytes requires expression within the cell of the proapoptotic $\mathrm{BH} 3$-only protein $\operatorname{Bim}(33,43)$. Levels of Bim protein are similar in resting and activated $\mathrm{T}$ cells; however, Bim drives rapid 
death only in the latter (33). Although the state of Bim might change between resting and activated cells, perhaps via changes in its phosphorylation state, its ability to drive activated $\mathrm{T}$ cell death is certainly not controlled by the amount of Bim protein expressed. Therefore, it is likely that the changes in levels of the $\mathrm{Bim}$ antagonist $\mathrm{Bcl}-2$ are important, and an understanding of the molecular mechanisms that control these changes are of critical importance to our understanding of ACAD.

\section{ROS and Bcl-2 expression}

As mentioned earlier, apoptosis of activated $T$ cells can be inhibited by culture with the antioxidant MnTBAP, indicating that ROS are somehow involved in ACAD (7). To find out whether the ROS caused T cell death by affecting gene transcription, we activated $\mathrm{T}$ cells in vivo, isolated the cells just before they were to die, and cultured them for a short time in the presence or absence of MnTBAP. mRNA was then isolated from the cells and its content compared using Affymetrix gene chips (Affymetrix Inc., Santa Clara, California, USA). The expression of many genes was changed by the presence of MnTBAP (data not shown). Interestingly, while expression of mRNA for Fas, FasL, and many of the proapoptotic Bcl-2 family members was unchanged, expression of the mRNA for $\mathrm{Bcl}-2$ itself was markedly increased by MnTBAP treatment (data not shown). These data strongly suggest that ROS can drive $\mathrm{Bcl}-2$ downregulation within activated T cells.

The transcription factor cAMP response elementbinding protein (CREB) is a potential target of these ROS, since ROS can decrease CREB function in other cell types (44) and CREB controls Bcl-2 levels in lymphoid cells (45) (Figure 3). In fact, a recent report has shown that, in neuronal cells, hypoxia (which can decrease ROS) can induce $\mathrm{Bcl}-2$, and that the cAMP response element is required for this inducibility (46). We are currently testing the possibility that ROS inactivation of CREB occurs in activated T cells and is responsible for the observed decrease in Bcl-2.

CREB may not be the only intermediate between ROS and loss of Bcl-2 mRNA. ROS affect the activity of NF- $\kappa B$, and NF- $\kappa B$ has been reported to increase mRNA levels of both Bcl-2 and its antiapoptotic relative $\mathrm{Bcl}-\mathrm{x}_{\mathrm{L}}$ (47). However, the reported effects of ROS on NF- $\mathrm{\kappa B}$ activity are confusing and contradictory. For example, ROS induce NF- $\mathrm{KB}$ activity by increasing degradation of $\mathrm{I} \kappa \mathrm{B}$ and consequently facilitating migration of NF- $\kappa B$ to the nucleus (48). On the other hand, ROS cause oxidation of a crucial cysteine residue at the DNA-binding site of NF- $\mathrm{\kappa B}$, thereby inhibiting the action of the transcription factor (49). Whether ROS activate or inhibit NF- $\mathrm{KB}$ activity is perhaps controlled by the amount and/or cellular location of the ROS.

Taken together, our results suggest a two-step model of ACAD. Signal one is provided by ROS-driven $\mathrm{Bcl}-2$ downregulation and is necessary, but not sufficient, for apoptosis. Evidence supporting this comes from the demonstration that activated T cells from Bim-defi- cient mice still have decreased levels of $\mathrm{Bcl}-2$ but do not die in vivo (33). Also, restoration of Bcl-2 levels via retrovirus can inhibit Bim-mediated apoptosis (33). Signal two, therefore, is provided by Bim. Since the levels of Bim do not change following $\mathrm{T}$ cell activation in vivo, factors underlying ROS-driven $\mathrm{Bcl}-2$ downregulation are of considerable interest.

\section{Other targets of ROS that might affect ACAD}

A huge number of papers have addressed the problem of which molecules are targets of the stress induced in cells by ROS. Besides the effects on Bcl-2 levels mentioned above, ROS may affect many other molecules potentially involved in ACAD. For example, ROS certainly oxidize membrane lipids, and these molecules, either in the cell or in mitochondrial membranes, could affect the ability of the cell to resist death. However, in preliminary experiments conducted in association with the laboratory of Robert Murphy, we have not found changes in lipid oxidation when comparing the membranes of resting, long-lived $\mathrm{T}$ cells with those of activated $T$ cells that are destined to die rapidly. Thus, it appears that the amount of ROS produced by $\mathrm{T}$ cells after activation is too small to mediate such lipid peroxidation and membrane destruction (data not shown).

ROS also act on proteins within the cell. Among these are many transcription factors. Loss of CREB and NF- $\kappa \mathrm{B}$ activity might reduce transcription of genes important to cell survival, in addition to those for $B c l-2$ and $B c l-x_{L}$, discussed above. Other targets of ROS, such as inhibitor-of-apoptosis proteins, SODs, and A1, may also be important. Changes in the activities of other transcription factors, including AP-1, nuclear factor $1, \mathrm{Sp} 1$, and various members of the forkhead and Stat families, may also be crucial $(50,51)$.

ROS also affect signal-transducing proteins. As discussed above, a number of groups have shown that PI3K and proteins downstream of the enzyme, such as $\mathrm{Akt} /$ protein kinase $\mathrm{B}$, are activated in cells treated with $\mathrm{H}_{2} \mathrm{O}_{2}$ or inducers of ROS. In the chicken pre-B cell line DT40, externally applied $\mathrm{H}_{2} \mathrm{O}_{2}$ increases intracellular $\mathrm{Ca}^{2+}$ levels via a pathway that includes Syk, Btk, the B cell linker protein BLNK, and phospholipase $C \gamma 2$ (52). Evidence suggests that Syk and/or Btk are the most upstream enzymes in this pathway. However, it is not known whether these proteins are the actual targets of chemical modification by $\mathrm{H}_{2} \mathrm{O}_{2}$. Obviously the downstream products, increased $\mathrm{Ca}^{2+}$ levels, and PI3K/Akt activation have many affects on lymphocytes, including, in the case of increased $\mathrm{Ca}^{2+}$, induction of FasL and increased sensitivity to AICD. Since Akt is often thought to act as a prosurvival rather than a death-inducing enzyme, it is not obvious how this portion of the ROS/PI3K pathway could contribute to the death of activated $T$ cells, unless the effects of Akt are outweighed by increased levels of $\mathrm{Ca}^{2+}$ in some undetermined way.

Other signal transduction proteins affected by ROS include the extracellular signal-regulated kinases (ERKs) 1 and 2 , and although ROS-induced activation of ERK2 is thought to be required for cell cycle progression (5), 
ROS may also affect Bcl-2 expression, as a cell cycleinhibitory effect of Bcl-2 has been described (12).

\section{Summary}

Thousands of studies of ROS and their effects on cells have been reported. Therefore it is not surprising that these powerful biological mediators play a role in the phenomena associated with $T$ cell activation and death. Even though activated $\mathrm{T}$ cells can die in two different ways, driven by external stimuli via AICD or by processes that are intrinsic to the cell via ACAD, evidence suggests that ROS are involved in both pathways. Although much of the signaling mediated by ROS in T cells remains unclear, the abundance of data on ROS signaling in nonlymphoid cell types should aid in the elucidation of ROS-driven signaling within T cells. Furthermore, since ROS are so crucial in determining the fates of activated $T$ cells, an understanding of how ROS achieve their effects may suggest therapeutic targets for the destruction of autoimmune $\mathrm{T}$ cells, and for the improvement of substandard vaccines - the twin goals of modern applied immunology.

Note added in proof. Reusch and colleagues recently showed that ROS specifically downregulated CREBdependent Bcl-2 promoter activity in primary neurons. (Pugazhenthi, S. et al. 2003. Oxidative stress-mediated down-regulation of $\mathrm{Bcl}-2$ promoter in hippocampal neurons. J. Neurochem. In press.)

\section{Acknowledgments}

This work was supported by US Public Health Service grants AI-17134, AI-18785, AI-22295, and AI-52225.

1. Badovinac, V.P., Porter, B.B., and Harty, J.T. 2002. Programmed contraction of CD8(+) T cells after infection. Nat. Immunol. 3:619-626.

2. Masopust, D., Vezys, V., Marzo, A.L., and Lefrancois, L. 2001. Preferential localization of effector memory cells in nonlymphoid tissue. Science. 291:2413-2417.

3. Reinhardt, R.L., Khoruts, A., Merica, R., Zell, T., and Jenkins, M.K. 2001 Visualizing the generation of memory CD4 T cells in the whole body. Nature. 410:101-105

4. Kawabi, Y., and Ochi, A. 1991. Programmed cell death and extrathymic reduction of Vbeta8 $+\mathrm{CD} 4+\mathrm{T}$ cells in mice tolerant to Staphylococcus aureus enterotoxin B. Nature. 349:245-248.

5. Goldstone, S.D., and Hunt, N.H. 1997. Redox regulation of the mitogenactivated protein kinase pathway during lymphocyte activation. Biochim. Biophys. Acta. 1355:353-360.

6. Devadas, S., Zaritskaya, L., Rhee, S.G., Oberley, L., and Williams, M.S 2002. Discrete generation of superoxide and hydrogen peroxide by $\mathrm{T}$ cell receptor stimulation: selective regulation of mitogen-activated protein kinase activation and fas ligand expression. J. Exp. Med. 195:59-70.

7. Hildeman, D.A., et al. 1999. Reactive oxygen species regulate activationinduced T cell apoptosis. Immunity. 10:735-744

8. Aulwurm, U.R., and Brand, K.A. 2000. Increased formation of reactive oxygen species due to glucose depletion in primary cultures of rat thymocytes inhibits proliferation. Eur. J. Biochem. 267:5693-5698.

9. Halliwell, B., and Gutteridge, J.M.C. 1985. Free radicals in biology and medicine. Oxford University Press. Oxford, United Kingdom. 189 pp.

10. Gerson, D.F., Kiefer, H., and Eufe, W. 1982. Intracellular pH of mitogenstimulated lymphocytes. Science. 216:1009-1010.

11. Harris, S.G., and Phipps, R.P. 2001. The nuclear receptor PPAR gamma is expressed by mouse T lymphocytes and PPAR gamma agonists induce apoptosis. Eur. J. Immunol. 31:1098-1105.

12. Huang, W.C., Chio, C.C., Chi, K.H., Wu, H.M., and Lin, W.W. 2002 Superoxide anion-dependent Raf/MEK/ERK activation by peroxisome proliferator activated receptor gamma agonists 15 -deoxy-delta $(12,14)$ prostaglandin J(2), ciglitazone, and GW1929. Exp. Cell Res. 277:192-200.

13. Cauley, L.S., Miller, E.E., Yen, M., and Swain, S.L. 2000. Superantigeninduced CD4 $\mathrm{T}$ cell tolerance mediated by myeloid cells and
IFN-gamma. J. Immunol. 165:6056-6066.

14. Gardner, P.R. 1997. Superoxide-driven aconitase Fe-S recycling. Biosci. Rep. 17:33-42.

15. Gardner, R., Salvador, A., and Moradas-Ferreira, P. 2002. Why does SOD overexpression sometimes enhance, sometimes decrease, hydrogen peroxide production? A minimalist explanation. Free Radic. Biol. Med. 32:1351-1357.

16. Droge, W., Eck, H.P., Gmunder, H., and Mihm, S. 1991. Modulation of lymphocyte functions and immune responses by cysteine and cysteine derivatives. Am. J. Med. 91(Suppl. 3C):140S-144S.

17. Ashwell, J.D., Cunningham, R.E., Noguchi, P.D., and Hernandez, D. 1987. Cell growth cycle block of $\mathrm{T}$ cell hybridomas upon activation with antigen. J. Exp. Med. 165:173-194.

18. Shi, Y.F., Sahai, B.M., and Green, D.R. 1989. Cyclosporin A inhibits activation-induced cell death in T-cell hybridomas and thymocytes. Nature. 339:625-626.

19. Van Parijs, L., Peterson, D.A., and Abbas, A.K. 1998. The Fas/Fas ligand pathway and $\mathrm{Bcl}-2$ regulate $\mathrm{T}$ cell responses to model self and foreign antigens. Immunity. 8:265-274.

20. Peter, M.E, and Krammer, P.H. 1998. Mechanisms of CD95 (APO-1/Fas)mediated apoptosis. Curr. Opin. Immunol. 10:545-551.

21. Tucek-Szabo, C.L., Andjelic, S., Lacy, E., Elkon, K.B., and Nikolic-Zugic, J. 1996. Surface T cell Fas receptor/CD95 regulation, in vivo activation, and apoptosis. Activation-induced death can occur without Fas receptor. J. Immunol. 156:192-200.

22. Tschopp, J., Irmler, M., and Thome, M. 1998. Inhibition of fas death signals by FLIPs. Curr. Opin. Immunol. 10:552-558.

23. Refaeli, Y., Van Parijs, L., London, C.A., Tschopp, J., and Abbas, A.K. 1998. Biochemical mechanisms of IL-2-regulated Fas-mediated T cell apoptosis. Immunity. 8:615-623.

24. Yeh, J.H., Hsu, S.C., Han, S.H., and Lai, M.Z. 1998. Mitogen-activated protein kinase kinase antagonized fas-associated death domain proteinmediated apoptosis by induced FLICE-inhibitory protein expression. J. Exp. Med. 188:1795-1802.

25. Chen, W., et al. 2001. Requirement for transforming growth factor beta 1 in controlling T cell apoptosis. J. Exp. Med. 194:439-453.

26. Schlapbach, R., et al. 2000. TGF-beta induces the expression of the FLICE-inhibitory protein and inhibits Fas-mediated apoptosis of microglia. Eur. J. Immunol. 30:3680-3688.

27. Ramsdell, F., et al. 1994. Differential ability of Th1 and Th2 T cells to express Fas ligand and to undergo activation-induced cell death. Int. Immunol. 6:1545-1553.

28. Williamson, J.R. 1986. Role of inositol lipid breakdown in the generation of intracellular signals. State of the art lecture. Hypertension. 8:II140-II156.

29. Schieven, G.L., et al. 1994. ZAP-70 tyrosine kinase, CD45, and T cell receptor involvement in $\mathrm{UV}$ - and $\mathrm{H} 2 \mathrm{O} 2$-induced $\mathrm{T}$ cell signal transduction. J. Biol. Chem. 269:20718-20726.

30. Rengarajan, J., et al. 2000. Sequential involvement of NFAT and Egr transcription factors in FasL regulation. Immunity. 12:293-300.

31. Bauer, M.K., et al. 1998. Role of reactive oxygen intermediates in activation-induced CD95 (APO-1/Fas) ligand expression. J. Biol. Chem. 273:8048-8055

32. Maccarone, M., Rosato, N., and Agro, A.F. 1995. Electroporation enhances cell membrane peroxidation and luminescence. Biochem. Biophys. Res. Commun. 206:238-245.

33. Hildeman, D.A., et al. 2002. Activated T cell death in vivo mediated by pro-apoptotic Bcl-2 family member Bim. Immunity. 16:759-767.

34. Gonzalo, J.A., et al. 1994. A single injection of Staphylococcus aureus enterotoxin B reduces autoimmunity in MRL/lpr mice. Clin. Immunol. Immunopathol. 71:176-182.

35. Hildeman, D.A., Zhu, Y., Mitchell, T., Kappler, J., and Marrack, P. 2002. Molecular mechanisms of activated $\mathrm{T}$ cell death in vivo. Curr. Opin. Immunol. 14:354-359.

36. Zajac, A.J., et al. 1998. Viral immune evasion due to persistence of activated T cells without effector function. J. Exp. Med. 188:2205-2213.

37. Newton, K., and Strasser, A. 1998. The Bcl-2 family and cell death regulation. Curr. Opin. Genet. Dev. 8:68-75.

38. Zong, W.X., Lindsten, T., Ross, A.J., MacGregor, G.R., and Thompson, C.B. 2001. BH3-only proteins that bind pro-survival Bcl-2 family members fail to induce apoptosis in the absence of Bax and Bak. Genes Dev. 15:1481-1486.

39. Martinou, J.C., and Green, D.R. 2001. Breaking the mitochondrial barrier. Nat. Rev. Mol. Cell Biol. 2:63-67.

40. Strasser, A., Harris, A.W., Huang, D.C., Krammer, P.H., and Cory, S. 1995. Bcl-2 and Fas/APO-1 regulate distinct pathways to lymphocyte apoptosis. EMBO J. 14:6136-6147.

41. Akbar, A.N., et al. 1993. The significance of low bcl-2 expression by $\mathrm{CD} 45 \mathrm{O} \mathrm{T}$ cells in normal individuals and patients with acute viral infections. The role of apoptosis in T cell memory. J. Exp. Med. 178:427-438.

42. Mitchell, T.C., et al. 2001. Immunological adjuvants promote activated T 
cell survival via induction of Bcl-3. Nat. Immunol. 2:397-402.

43. Bouillet, P., et al. 2002. BH3-only Bcl-2 family member Bim is required for apoptosis of autoreactive thymocytes. Nature. 415:922-926.

44. Watson, P.A., Nesterova, A., Burant, C.F., Klemm, D.J., and Reusch, J.E. 2001. Diabetes-related changes in cAMP response element-binding protein content enhance smooth muscle cell proliferation and migration. J. Biol. Chem. 276:46142-46150.

45. Wilson, B.E., Mochon, E., and Boxer, L.M. 1996. Induction of bcl-2 expression by phosphorylated CREB proteins during B-cell activation and rescue from apoptosis. Mol. Cell. Biol. 16:5546-5556.

46. Freeland, K., Boxer, L.M., and Latchman, D.S. 2001. The cyclic AMP response element in the $\mathrm{Bcl}-2$ promoter confers inducibility by hypoxia in neuronal cells. Brain Res. Mol. Brain Res. 92:98-106.

47. Bureau, F., et al. 2002. Constitutive nuclear factor-kappaB activity preserves homeostasis of quiescent mature lymphocytes and granulocytes by controlling the expression of distinct $\mathrm{Bcl}-2$ family proteins. Blood. 99:3683-3691.

48. Majumdar, S., Lamothe, B., and Aggarwal, B.B. 2002. Thalidomide suppresses NF-kappa B activation induced by TNF and $\mathrm{H} 2 \mathrm{O} 2$, but not that activated by ceramide, lipopolysaccharides, or phorbol ester. J. Immunol. 168:2644-2651

49. Ginis, I., et al. 2000. Tumor necrosis factor and reactive oxygen species cooperative cytotoxicity is mediated via inhibition of NF-kappaB. Mol. Med. 6:1028-1041.

50. Hirota, K., et al. 1997. AP-1 transcriptional activity is regulated by a direct association between thioredoxin and Ref-1. Proc. Natl. Acad. Sci. U. S. A. 94:3633-3638.

51. Waris, G., Huh, K.W., and Siddiqui, A. 2001. Mitochondrially associated hepatitis $\mathrm{B}$ virus $\mathrm{X}$ protein constitutively activates transcription factors STAT-3 and NF-kappa B via oxidative stress. Mol. Cell. Biol. 21:7721-7730.

52. Qin, S., and Chock, P.B. 2001. Bruton's tyrosine kinase is essential for hydrogen peroxide-induced calcium signaling. Biochemistry. 40:8085-8091.

53. Maxwell, J.R., Weinberg, A., Prell, R.A., and Vella, A.T. 2000. Danger and OX40 receptor signaling synergize to enhance memory $\mathrm{T}$ cell survival by inhibiting peripheral deletion. J. Immunol. 164:107-112.

54. Mitchell, T., Kappler, J., and Marrack, P. 1999. Bystander virus infection prolongs activated T cell survival. J. Immunol. 162:4527-4535. 